\title{
Poor prognostic factors in human papillomavi- rus-positive head and neck cancer: who might not be candidates for de-escalation treatment?
}

\author{
Shin Hye Yoo ${ }^{1}$, Chan-Young Ockㄹ, Bhumsuk Keam ${ }^{1}$, Sung Joon Park ${ }^{2}$, Tae Min Kim¹, Jin Ho Kim³, \\ Yoon Kyung Jeon ${ }^{4}$, Eun-Jae Chung ${ }^{2}$, Seong Keun Kwon ${ }^{2}$, J. Hun $\mathrm{Hah}^{2}$, Tack-Kyun Kwon ${ }^{2}$, \\ Kyeong Chun Jung ${ }^{4}$, Dong-Wan Kim ${ }^{1}$, Hong-Gyun Wu ${ }^{3}$, Myung-Whun Sung ${ }^{2}$, and Dae Seog Heo
}

Departments of ${ }^{1}$ Internal Medicine, ${ }^{2}$ Otorhinolaryngology, ${ }^{3}$ Radiation Oncology, and ${ }^{4}$ Pathology, Seoul National University Hospital, Seoul, Korea

Received: November 29, 2017 Revised : April 6, 2018 Accepted: May 2, 2018

\section{Correspondence to}

Bhumsuk Keam, M.D.

Department of Internal

Medicine, Seoul National

University Hospital, 101

Daehak-ro, Jongno-gu, Seoul

03080, Korea

Tel: +82-2-2072-7215

Fax: +82-2-2072-7379

E-mail: bhumsuk@snu.ac.kr

This study was presented in part at the 52th Annual Meeting of the American Society of Clinical Oncology, Chicago, IL, June 3 to 7, 2016.
Background/Aims: Since patients with human papillomavirus (HPV)-associated head and neck squamous cell carcinoma (HNSCC) have favorable outcomes after treatment, treatment de-escalation for these patients is being actively investigated. However, not all HPV-positive HNSCCs are curable, and some patients have a poor prognosis. The purpose of this study was to identify poor prognostic factors in patients with HPV-positive HNSCC.

Methods: Patients who received a diagnosis of HNSCC and tested positive for HPV from 2000 to 2015 at a single hospital site $(n=152)$ were included in this retrospective analysis. HPV typing was conducted using the HPV DNA chip assay or liquid bead microarray system. Expression of p16 in the tumors was assessed by immunohistochemistry. To determine candidate factors associated with overall survival (OS), univariate and multivariable Cox regression analyses were performed.

Results: A total of 152 patients with HPV-positive HNSCC were included in this study; $82.2 \%$ were male, $43.4 \%$ were current or former smokers, and $84.2 \%$ had oropharyngeal cancer. By univariate analysis, old age, performance status $\geq 1$, non-oropharyngeal location, advanced T classification ( $\left.\mathrm{T}_{3}-4\right)$, and HPV genotype 18 were significantly associated with poor OS. By multivariable analysis, performance status $\geq 1$ and non-oropharyngeal location were independently associated with shorter OS (hazard ratio [HR], 4.36, $p=0.015$; HR, 11.83, $p=0.002$, respectively). Furthermore, HPV genotype 18 positivity was also an independent poor prognostic factor of OS (HR, 10.87, $p<0.001)$.

Conclusions: Non-oropharyngeal cancer, poor performance status, and HPV genotype 18 were independent poor prognostic factors in patients with HPV-positive HNSCC. Patients with these risk factors might not be candidates for de-escalation treatment.

Keywords: Human papillomavirus; Head and neck neoplasms; Overall survival; De-escalation 


\section{INTRODUCTION}

Head and neck cancer ranks seventh highest in prevalence among Korean males, and adds 16.7 patients per 100,000 annually to the overall cancer burden in Korea [1]. It is well-known that human papillomavirus (HPV) is a causative factor for head and neck squamous cell carcinoma (HNSCC), along with smoking and heavy alcohol consumption. HPV infection is associated with the development of oropharyngeal cancers (OPCs). Several studies, including a large meta-analysis, have reported that patients with HPV-positive HNSCC have better overall survival (OS) and disease-free survival (DFS) than patients with HPV-negative HNSCC $[2,3]$. Since the 5-year OS of HPV-positive HNSCC patients is approximately $80 \%$ to $90 \%$, the use of de-escalation treatment for these patients is being investigated [4]. The goal of de-escalation treatment is to maintain proper cure rates and to minimize long-term morbidity [4]. Various approaches to less intensive treatment are being evaluated in clinical trials, including the following: (1) replacing cisplatin with an anti-epidermal growth factor receptor agent such as cetuximab; (2) decreasing the radiation dose; and (3) performing minimally invasive surgery such as transoral microendoscopic laser surgery.

However, despite these de-escalation attempts, the current practice guidelines do not recommend de-escalation treatment for HPV-positive HNSCC patients due to lack of evidence [5]. In addition, despite the overall favorable outcomes of patients with HPV-positive OPC, some patients still relapse. To date, little is understood about the clinical and molecular features associated with poor outcomes in HPV-positive HNSCC patients, although a previous retrospective analysis found that patients with HPV-positive OPC who smoked for more than 10 pack years and had N2b-N3 disease had a 5-year survival of approximately $60 \%$ [3]. Hence, we hypothesized that some HPV-positive HNSCC patients should not be candidates for treatment de-escalation. The aim of our study was to identify poor prognostic factors which may affect OS or DFS in HPV-positive HNSCC patients.

\section{METHODS}

\section{Patient selection and collection of clinical data}

Records of patients diagnosed with HNSCC at Seoul National University Hospital from January 2000 to February 2015 were retrospectively analyzed using an electronic database. Inclusion criteria were as follows: patients (1) with pathologically confirmed, locally advanced HNSCC (stage I-III or stage IV Mo); (2) treated with surgery, concurrent chemoradiation therapy (CCRT), or radiation therapy (RT); (3) older than 19 years at the time of diagnosis; and (4) with positive tests for HPV by liquid-bead microarray or DNA chip technology. Patients with an initial diagnosis of stage IV Mr disease or nasopharyngeal cancer were excluded. We investigated the location of primary tumor (oropharynx, hypopharynx, larynx, oral cavity, nasal cavity, or salivary gland) and the initial pathology (undifferentiated, poorly differentiated, moderately differentiated, or miscellaneous). Other baseline demographics (age, gender, smoking history, alcohol intake) and clinical data (Eastern Cooperative Oncology Group [ECOG] performance status [PS], and comorbidities including diabetes mellitus, hypertension, ischemic heart disease, liver cirrhosis, chronic obstructive pulmonary disease, and brain hemorrhage or stroke) were also obtained. We followed the guidelines outlined in the seventh edition of the American Joint Committee on Cancer to determine tumor staging [6]. All procedures involving human participants were performed in accordance with the ethical standards of the institutional and/or national research committees and with the 1964 Declaration of Helsinki and its later amendments, or comparable ethical standards. This study was approved by the Institutional Review Board of Seoul National University Hospital (approval number: $\mathrm{H}-1508-083-695)$. Due to the retrospective nature of this study, patients' consent to participate was waived in accordance with the Institutional Review Board.

\section{Treatment and response evaluation}

We retrospectively reviewed the electronic medical chart to determine whether induction treatment was present and the type of regimens used, the type of definitive treatment (surgery or non-surgery [CCRT or RT]), and adjuvant treatment modalities (CCRT, RT). The protocol for RT or CCRT in this cohort was described in prior 
reports $[7,8]$. Briefly, among the CCRT group, patients were directly given fractionated radiotherapy of more than 60 Gy for primary tumors and regional lymph nodes with concurrent use of chemotherapeutic agents (cisplatin, carboplatin, or cetuximab). The RT group followed the same protocol as that of the CCRT group, except that concurrent chemotherapy was not used.

We also evaluated the tumor response to induction treatment and definitive treatment using the Response Evaluation Criteria in Solid Tumors (version 1.1) [9] and calculated the objective response rate (ORR) as the percentage of cases with a complete response (CR) or a partial response among all the patients whose tumor responses we were able to evaluate. During follow-up care, the disease status after the response to definitive treatment was classified into three categories: (1) relapse, defined as any evidence of disease recurrence after CR; (2) progression in non-CR, defined as disease progression when a patient did not achieve CR to definitive treatment; and (3) no relapse nor progression, defined as continued lack of evidence of disease. We also reviewed the site of relapse, including locoregional or distance relapse. If the relapsed disease was only limited to the area within the head and neck and adjacent structures including lymph nodes, the relapse was defined as a locoregional relapse, and the remaining were defined as distance relapse.

\section{Tests for HPV infection}

Tumor specimens were fixed in formalin and embedded in paraffin. After DNA was extracted from a processed specimen, either of the following HPV genotyping methods were used: (1) HPV DNA Chip [10,11] or (2) HPV Liquid Bead Microarray [12].

The HPV DNA Chip is a polymerase chain reaction (PCR)-based DNA microarray system (MyGene, Seoul, Korea). Details on the methods performed after amplification were described previously [11]. Briefly, 24 type-specific probes were utilized for HPV genotyping: 15 highrisk (HR) genotypes $(16,18,31,33,35,39,45,51,52,53,56,58$, 59,66 , and 68) and nine low-risk (LR) genotypes (6, 11, 34, 40, 42, 43, 44, 54, and 70). Samples appearing as a positive 150-bp band on gel electrophoresis but negative on the HPV DNA chip slide were designated as HPV-other.

The HPV Liquid Bead Microarray was performed using a PCR cycler and a Luminex analyzer (GeneFinder, Infopia Inc., Anyang, Korea). Hybridization of amplified
DNA to probes coupled with beads in the Genefinder HPV Liquid Bead Microarray kit was performed according to the manufacturers' recommendations. Details of this process have been described previously [12]. This method detects 32 kinds of HPV genotypes simultaneously: 18 HR types $(16,18,26,31,33,35,39,45,51,52,53,56$, 58, 59, 66, 68, 69, and 73) and 14 LR types $(6,11,32,34,40$, 42, 43, 44, 54, 55, 62, 70, 81, and 83). An HPV copy number $\geq 100$ was designated as HPV-positive. The proportions of specimens positive for HPV genotypes 16 and 18 were determined, and other HR HPV genotypes were noted when present. Any sample positive for a HR genotype was defined as a HR type, and any sample positive for only a LR genotype was defined as a LR type.

\section{Immunohistochemistry}

Fixed and paraffin-embedded samples were evaluated by $\mathrm{p}_{1} 6^{\mathrm{INK}} 4 \mathrm{a}$ immunohistochemistry (IHC) using clone E6H4 of CINtec (Roche, Heidelberg, Germany) to determine the p16 status of a tumor specimen. The sample was considered positive for $\mathrm{p} 16$ if diffuse nuclear and cytoplasmic staining were present, and $>70 \%$ of cells were stained.

\section{Statistical analysis}

The primary outcome was OS, which was defined as the time from the date of diagnosis to the date of death, or last follow-up if censored, which was estimated using the Kaplan-Meier method. DFS was the secondary outcome, which was defined as the time from the date of diagnosis to the date of first confirmation of relapse or progression, also estimated by the Kaplan-Meier method. The independent variables used to construct a univariate logistic regression model for prognosis were demographic factors, disease-specific factors, HPV genotypes 16 and 18, and p16 status. Factors associated with OS and DFS were analyzed by univariate and multivariable Cox regression analyses, except for variables that did not satisfy the Cox proportional hazard assumption (nodal classification, definitive treatment, and the type of HPV testing method). If these variables were statistically significant in univariate analysis, they were included into multivariable model by stratification. Statistical significance was defined as $p<0.05$. All statistical tests were two-sided and were carried out using STATA software version 12 (StataCorp LP, College Station, TX, USA). 


\section{RESULTS}

\section{Baseline patient characteristics}

The 152 HPV-positive HNSCC study patients consisted of 125 men and 27 women. The proportion of patients who were current or former smokers at the time of diagnosis was $43.4 \%$, and the proportion of patients with ECOG PS O was $52.6 \%(n=80)$. The types of primary HPV-positive HNSCC included 129 OPCs, five hypopharyngeal cancers, one laryngeal cancer, 17 cancers of the oral cavity, and one salivary gland squamous cell carcinoma.

The baseline characteristics of the study population are shown in Table 1 . The positivity rates of HPV genotypes 16 and 18 were $73 \%$ and $14.5 \%$, respectively. The number of patients positive for both HPV genotypes 16 and 18 was six (4.0\%). Excluding 47 patients whose specimens were not assessed by p16 IHC, 23 patients were negative for p16. The prevalence of HR HPV genotypes was significantly lower in non-OPCs than in OPCs $(8.2 \%$ vs. $91.5 \%$, respectively; $p<0.001$ ). The prevalence of HPV genotype 16 was significantly lower in non-OPCs as well (6.3\% vs. 93.7\%, respectively; $p<0.001$ ).

Among 67 patients in the primary surgery group, 20 patients (29.9\%) underwent CCRT and 34 patients (19.4\%) underwent RT as adjuvant treatment. Only 13 patients (19.4\%) had no adjuvant treatment. None of the patients included in the study received treatment de-escalation.

\section{Overall and disease-free survival}

During median follow-up period of 29.5 months, 26 of 152 patients $(16.5 \%)$ died. The 5-year OS was $98.0 \%$ and DFS was $92.6 \%$. The ORR to the definitive treatments was $95.4 \%(n=145)$. There were no differences between the ORRs, OSs, and DFSs of the patients with HR HPV and those with LR HPV (Table 2). However, 32 of 135 patients (21.1\%) who achieved CR later relapsed. Locoregional recurrence was the most common type of relapse.

\section{Survival analysis using demographic and clinical factors}

We analyzed demographic factors, disease-specific factors, HPV genotypes 16 and 18, and p16 status to determine whether these affect OS or DFS. Table 3 shows the results of the univariate and multivariable Cox regression analyses of these factors in relation to OS (Fig. 1) and DFS (Fig. 2). Multivariable analysis of these factors identified PS, location of primary tumor, and HPV genotype 18 as significant predictors of shortened OS and DFS, with stratification of nodal classification (No vs. $\mathrm{N}_{1}-\mathrm{N}_{3}$ ), definitive treatment (surgery vs. non-surgery), and type of HPV testing method (liquid microarray vs. DNA chip). Similar results were observed when smoking was included, and it may be a relevant confounding factor even though it does not reach significance in univariate analysis or multivariable analysis (data not shown). p16 status was significant by univariate analysis but was excluded from further analysis because of its collinearity.

\section{DISCUSSION}

In this study, HPV-positive HNSCC had a CR rate exceeding $85 \%$, and $70 \%$ of these patients continued to show no evidence of disease over the follow-up period. However, we found that patients with HPV genotype 18 infection, non-OPC, and poor PS relapsed and died earlier than patients without these prognostic factors.

HPV genotype 18 positivity was independently associated with poor outcome. Whereas the carcinogenicity of HPV genotype 16 is well established, the roles of the HR HPV genotype 18 in OPC and non-OPC are unclear. Single infections with HPV genotype 18 and coinfection by HPV genotypes 16 and 18 were found to be relatively less prevalent in OPCs than in non-OPCs in our study, as well as in a previous systematic review [13]. On the other hand, HPV genotype 18 infections are known to be associated with aggressive disease and rapid progression [14]. Moreover, HPV genotype 18 appears to have a different molecular expression pattern, which suggests a pathway to carcinogenesis different from that of HPV genotype 16 [14]. Recent reports demonstrated that immune-related markers such as PD-Li expression on tumor cells or immune cells may affect survival in $\mathrm{HN}$ SCC [15]. However, there have been no definitive studies demonstrating that HPV genotype 18-positive HNSCC patients have different tumor immune infiltrates than HPV 16-positive HNSCC patients. According to a study comparing the survival and characteristics of HPV genotype 16 and other HPV genotypes [16], HPV genotype 16 comprised $84 \%$ of 73 HPV-positive HNSCC samples. Among the 12 remaining samples harboring other HPV 
Table 1. Baseline characteristics of HPV-positive patients with head and neck squamous cell carcinoma $(n=152)$

\begin{tabular}{|c|c|}
\hline Variable & No. (\%) \\
\hline Age at diagnosis, median (range) & $58(29-83)$ \\
\hline \multicolumn{2}{|l|}{ Gender } \\
\hline Male & $125(82.2)$ \\
\hline Female & $27(17.8)$ \\
\hline \multicolumn{2}{|l|}{ Smoking status at diagnosis } \\
\hline Current & $19(12.5)$ \\
\hline Ex-smoker & $47(30.9)$ \\
\hline Never smoker & $75(49 \cdot 3)$ \\
\hline Unknown & $11(7.2)$ \\
\hline No. of pack-year, mean (range) & $10.9(0-55)$ \\
\hline$\geq 10$ pack-year & $46(33.8)$ \\
\hline$<10$ pack-year & $90(66.2)$ \\
\hline Alcohol (no. per week), mean (range) & $0.3(0-5)$ \\
\hline Unknown & $7(4.6)$ \\
\hline \multicolumn{2}{|l|}{ Comorbidity $^{\mathrm{a}}$} \\
\hline Yes & $62(40.8)$ \\
\hline No & $90(59.2)$ \\
\hline \multicolumn{2}{|l|}{ ECOG PS } \\
\hline $\mathrm{O}$ & $80(52.6)$ \\
\hline 1 & $66(43 \cdot 4)$ \\
\hline 2 & $6(4.0)$ \\
\hline \multicolumn{2}{|l|}{ Primary tumor location } \\
\hline Oropharynx & $129(84.8)$ \\
\hline Hypopharynx & $5(3 \cdot 3)$ \\
\hline Larynx & $1(0.7)$ \\
\hline Oral cavity & $16(10.5)$ \\
\hline Others $^{\mathrm{b}}$ & $1(0.7)$ \\
\hline \multicolumn{2}{|l|}{ Staging } \\
\hline \multicolumn{2}{|l|}{ Tumor classification } \\
\hline To & $3(2.0)$ \\
\hline $\mathrm{T} 1$ & $36(24.0)$ \\
\hline $\mathrm{T} 2$ & $72(48.0)$ \\
\hline $\mathrm{T}_{3}$ & $18(12.0)$ \\
\hline $\mathrm{T}_{4}$ & $21(14.0)$ \\
\hline \multicolumn{2}{|l|}{ Nodal classification } \\
\hline No & $20(13 \cdot 3)$ \\
\hline $\mathrm{N} 1$ & $21(14.0)$ \\
\hline $\mathrm{N} 2$ & $103(68.7)$ \\
\hline $\mathrm{N}_{3}$ & $6(4.0)$ \\
\hline \multicolumn{2}{|l|}{ TNM stage } \\
\hline I-III & $38(25 \cdot 4)$ \\
\hline IV & $112(74.7)$ \\
\hline \multicolumn{2}{|l|}{ Pathology } \\
\hline Well differentiated & $17(11.2)$ \\
\hline
\end{tabular}

Table 1. Continued

\begin{tabular}{|c|c|}
\hline Variable & No. (\%) \\
\hline Moderately differentiated & $56(36.8)$ \\
\hline Poorly differentiated & $33(21.7)$ \\
\hline Others $^{c}$ & $46(30.3)$ \\
\hline \multicolumn{2}{|l|}{ Induction chemotherapy } \\
\hline Received & $52(34.2)$ \\
\hline Not received & $100(65.8)$ \\
\hline \multicolumn{2}{|l|}{ Definitive treatment } \\
\hline Radical CCRT & $68(44.7)$ \\
\hline Radical RT & $16(10.5)$ \\
\hline Surgery only & $13(8.5)$ \\
\hline Surgery followed by RT & $34(22.4)$ \\
\hline Surgery followed by CCRT & $20(13.2)$ \\
\hline Palliative chemotherapy & $1(0.7)$ \\
\hline \multicolumn{2}{|l|}{ HPV test method } \\
\hline Liquid-bead microarray & $57(37.5)$ \\
\hline DNA chip & $95(62.5)$ \\
\hline \multicolumn{2}{|l|}{ HPV genotyping } \\
\hline \multicolumn{2}{|l|}{ HPV genotype 16} \\
\hline Positive & $111(73.0)$ \\
\hline Negative & $41(27.0)$ \\
\hline \multicolumn{2}{|l|}{ HPV genotype 18} \\
\hline Positive & $22(14 \cdot 5)$ \\
\hline Negative & $130(85.5)$ \\
\hline \multicolumn{2}{|l|}{ HPV genotype ${ }^{\mathrm{d}}$} \\
\hline High-risk & $134(88.2)$ \\
\hline Low-risk only & $11(7.2)$ \\
\hline Unknown & $7(4.6)$ \\
\hline \multicolumn{2}{|l|}{ p16 status } \\
\hline Strong positive & $82(54.0)$ \\
\hline Negative & $23(15.1)$ \\
\hline Not tested (unknown) & $47(30.9)$ \\
\hline
\end{tabular}

HPV, human papillomavirus; ECOG, Eastern Cooperative Oncology Group; PS, performance status; TNM, tumour, node, metastasis; CCRT, concurrent chemoradiation therapy; $\mathrm{RT}$, radiation therapy.

${ }^{\mathrm{a} C o m o r b i d i t i e s ~ i n c l u d e s ~ d i a b e t e s ~ m e l l i t u s, ~ h y p e r t e n s i o n, ~}$ ischemic heart disease, liver cirrhosis, chronic obstructive pulmonary disease, brain hemorrhage or stroke.

${ }^{\mathrm{b}}$ Other site was salivary gland cancer with pathologic type of squamous cell carcinoma.

${ }^{\mathrm{c}}$ Other pathologic types consist of undifferentiated type, nonkeratinizing type, not otherwise specified, and unknown type.

${ }^{\mathrm{d}}$ Multiple HPV infections ( $\mathrm{n}=21,13.8 \%$ ) of 152 study patients. 
Table 2. Clinical outcomes according to high- and low-risk HPV genotypes of the tumors of patients with HPV-positive HNSCCs

\begin{tabular}{|c|c|c|c|c|}
\hline Variable & HPV-positive HNSCC & $\begin{array}{l}\text { HR HPV } \\
(\mathrm{n}=134)\end{array}$ & $\begin{array}{l}\text { LR HPV } \\
(\mathrm{n}=11)\end{array}$ & $p$ value \\
\hline \multicolumn{5}{|l|}{ Response to induction chemotherapy } \\
\hline $\mathrm{CR} / \mathrm{nCR}$ & $8(15 \cdot 7)$ & $6(13.0)$ & o & 0.229 \\
\hline $\mathrm{PR}$ & $30(58.8)$ & $28(60.9)$ & $1(50.0)$ & \\
\hline $\mathrm{SD}$ & $6(11.8)$ & $6(13.0)$ & o & \\
\hline PD & $4(7.8)$ & $4(8.7)$ & o & \\
\hline $\mathrm{NE}$ & $3(5 \cdot 9)$ & $2(4 \cdot 4)$ & $1(50.0)$ & \\
\hline \multicolumn{5}{|l|}{ Response to treatment } \\
\hline $\mathrm{CR} / \mathrm{nCR}$ & $135(88.8)$ & $119(88.8)$ & $9(81.8)$ & 0.218 \\
\hline PR & $10(6.6)$ & $9(6.7)$ & $1(9.1)$ & \\
\hline $\mathrm{SD}$ & 0 & o & o & \\
\hline $\mathrm{PD}$ & $4(2.6)$ & $4(3.0)$ & o & \\
\hline $\mathrm{NE}$ & $3(2.0)$ & $2(1.5)$ & $1(9.1)$ & \\
\hline \multicolumn{5}{|l|}{ Disease status } \\
\hline Relapse & $32(21.1)$ & $27(20.12)$ & $3(27 \cdot 3)$ & 0.769 \\
\hline Progression in non-CR & $12(7 \cdot 9)$ & $11(8.2)$ & $1(9.1)$ & \\
\hline No relapse nor progression & $108(71.0)$ & $96(71.6)$ & $7(63.6)$ & \\
\hline \multicolumn{5}{|l|}{ Relapse site } \\
\hline Locoregional & $25(16.7)$ & $20(15.2)$ & $4(36.4)$ & 0.482 \\
\hline Distant & $8(5 \cdot 3)$ & $7(5 \cdot 3)$ & $\mathrm{O}$ & \\
\hline Both & $4(2.7)$ & $4(3.0)$ & o & \\
\hline Death & $6(4 \cdot 0)$ & $6(4 \cdot 5)$ & $\mathrm{O}$ & \\
\hline None & $107(71.3)$ & $95(72.0)$ & $7(63.6)$ & \\
\hline Disease-free survival, mon, median (95\% CI) & $\mathrm{NR}(75 \cdot 0-\mathrm{NR})$ & $\mathrm{NR}(75 \cdot 0-\mathrm{NR})$ & $44 \cdot 4(9 \cdot 3-\mathrm{NR})$ & 0.286 \\
\hline \multicolumn{5}{|l|}{ Death } \\
\hline Yes & $26(17.1)$ & $23(17.2)$ & $1(9.1)$ & 0.692 \\
\hline No (censored) & $126(82.9)$ & $111(82.8)$ & $10(90.9)$ & \\
\hline Overall survival, mon, median (95\% CI) & NR (115.8-NR) & NR (12.6-NR) & NR $(115.8-N R)$ & 0.753 \\
\hline Follow-up duration, mon, median (range) & $29.5(2.2-205.9)$ & $30.3(2.2-205.9)$ & $21.3(9.4-73.7)$ & \\
\hline
\end{tabular}

Values are presented as number (\%) unless otherwise indicated.

HPV, human papillomavirus; HNSCC, head and neck squamous cell carcinoma; HR, high-risk; LR, low-risk; CR, complete response; nCR, near complete response; PR, partial response; SD, stable disease; PD, progressive disease; NE, not evaluable; NR, not reached; CI, confidence interval.

genotypes, there were eight HPV genotype 33, three HPV genotype 35, and one HPV genotype 56, and none of these samples were HPV genotype 18-postive. Several viral gene expressions differed between HPV genotype 16 and the other HPV-infected cohort, although the clinical characteristics and genomic aberration were similarly distributed. In addition, the other HPV-infected cohort had better 3-year OS than the HPV genotype
16 cohort. Taken together, these findings suggest that different levels of viral gene expression induced by HPV genotypes other than HPV genotype 16 might influence patient survival. Although limited data exist for HPV genotype 18 , it is possible that similar mechanistic links may be present in correlation with this genotype. Further research is warranted to investigate the immune infiltrates in HPV genotype 18-positive HNSCC patients. 
Yoo SH, et al. Poor prognostic factors in HPV positive head and neck cancer

Table 3. Cox univariate and multivariable analysis for overall and disease-free survival of patients with HPV-positive HNSCC

\begin{tabular}{|c|c|c|c|c|c|c|c|c|}
\hline \multirow{3}{*}{ Variable } & \multicolumn{4}{|c|}{ Overall survival } & \multicolumn{4}{|c|}{ Disease-free survival } \\
\hline & \multicolumn{2}{|c|}{ Univariate } & \multicolumn{2}{|c|}{ Multivariable } & \multicolumn{2}{|c|}{ Univariate } & \multicolumn{2}{|c|}{ Multivariable } \\
\hline & $\begin{array}{l}\text { Hazard ratio } \\
(95 \% \mathrm{CI})\end{array}$ & $p$ value & $\begin{array}{l}\text { Hazard ratio } \\
(95 \% \mathrm{CI})\end{array}$ & $p$ value & $\begin{array}{l}\text { Hazard ratio } \\
\quad(95 \% \mathrm{CI})\end{array}$ & $p$ value & $\begin{array}{l}\text { Hazard ratio } \\
(95 \% \mathrm{CI})\end{array}$ & $p$ value \\
\hline Age at diagnosis & $1.05(1.01-1.09)$ & 0.009 & $1.03(0.98-1.09)$ & 0.292 & $1.05(1.01-1.08)$ & 0.005 & $0.99(0.94-1.04)$ & 0.681 \\
\hline \multicolumn{9}{|l|}{ Gender } \\
\hline Female & $1.00(\mathrm{ref})$ & & NI & & $1.00(\mathrm{ref})$ & & NI & \\
\hline Male & $1.24(0.43-3.60)$ & 0.695 & & & $1.53(0.53-4.38)$ & 0.428 & & \\
\hline \multicolumn{9}{|l|}{ Smoking status ${ }^{\mathrm{a}}$} \\
\hline <10 pack-year & 1.00 (ref) & & NI & & $1.00(\mathrm{ref})$ & & NI & \\
\hline$\geq 10$ pack-year & $1.53(0.68-3.42)$ & 0.301 & & & $1.59(0.77-3.26)$ & 0.206 & & \\
\hline \multicolumn{9}{|c|}{ Alcohol (no. per week) } \\
\hline No & $1.00(\mathrm{ref})$ & & NI & & $1.00(\mathrm{ref})$ & & NI & \\
\hline Yes & $1.73(0.73-4.09)$ & 0.210 & & & $1.27(0.56-2.87)$ & 0.563 & & \\
\hline \multicolumn{9}{|l|}{ Comorbidity } \\
\hline No & 1.00 (ref) & & NI & & 1.00 (ref) & & NI & \\
\hline Yes & $1.34(0.60-2.95)$ & 0.475 & & & $0.97(0.45-2.10)$ & 0.936 & & \\
\hline \multicolumn{9}{|l|}{ ECOG PS } \\
\hline 0 & 1.00 (ref) & & 1.00 (ref) & & 1.00 (ref) & & 1.00 (ref) & \\
\hline$\geq 1$ & $3.10(1.28-7.46)$ & 0.012 & $4.36(1.33-14.25)$ & 0.015 & $2.90(1.32-6.35)$ & 0.008 & $3.00(1.07-8.43)$ & 0.037 \\
\hline \multicolumn{9}{|c|}{ Primary tumor location } \\
\hline Oropharynx & $1.00(\mathrm{ref})$ & & 1.00 (ref) & & 1.00 (ref) & & $1.00(\mathrm{ref})$ & \\
\hline $\begin{array}{l}\text { Non- } \\
\text { oropharynx }\end{array}$ & $2.98(1.16-7.66)$ & 0.023 & $11.83(2.57-54.43)$ & 0.002 & $2.98(1.26-7.08)$ & 0.013 & $3.72(1.02-13.54)$ & 0.046 \\
\hline \multicolumn{9}{|c|}{ Tumor classification } \\
\hline To-T2 & $1.00(\mathrm{ref})$ & & 1.00 (ref) & & $1.00(\mathrm{ref})$ & & $1.00(\mathrm{ref})$ & \\
\hline $\mathrm{T}_{3}-\mathrm{T}_{4}$ & $3.16(1.44-6.95)$ & 0.004 & $2.11(0.76-5.82)$ & 0.151 & $2.64(1.26-5.53)$ & 0.010 & $1.58(0.61-4.13)$ & 0.349 \\
\hline \multicolumn{9}{|l|}{ TNM stage } \\
\hline I-III & 1.00 (ref) & & NI & & $1.00(\mathrm{ref})$ & & NI & \\
\hline IV & $0.97(0.39-2.42)$ & 0.941 & & & $0.55(0.26-1.16)$ & 0.115 & & \\
\hline \multicolumn{9}{|l|}{ Pathology } \\
\hline Non-PD & $1.00(\mathrm{ref})$ & & NI & & $1.00(\mathrm{ref})$ & & NI & \\
\hline $\mathrm{PD}$ & $0.49(0.15-1.62)$ & 0.241 & & & $0.61(0.23-1.59)$ & 0.308 & & \\
\hline \multicolumn{9}{|l|}{ HPV genotype } \\
\hline $\begin{array}{l}\text { HPV genotype } \\
16 \text { positive }\end{array}$ & 1.00 (ref) & & 1.00 (ref) & & 1.00 (ref) & & 1.00 (ref) & \\
\hline $\begin{array}{l}\text { HPV genotype } \\
16 \text { negative }\end{array}$ & $3.89(1.77-8.52)$ & 0.001 & $0.96(0.29-3.21)$ & 0.952 & $2.01(0.98-4.12)$ & 0.057 & $0.52(0.15-1.80)$ & 0.304 \\
\hline $\begin{array}{l}\text { HPV genotype } \\
18 \text { negative }\end{array}$ & 1.00 (ref) & & 1.00 (ref) & & $1.00(\mathrm{ref})$ & & $1.00(\mathrm{ref})$ & \\
\hline $\begin{array}{l}\text { HPV genotype } \\
18 \text { positive }\end{array}$ & $5.06(2.32-11.02)$ & $<0.001$ & $10.87(3.06-38.57)$ & $<0.001$ & $3.26(1.55-6.86)$ & 0.002 & $7.38(2.02-26.92)$ & 0.002 \\
\hline HR type & & & & & & & & \\
\hline
\end{tabular}


Table 3. Continued

\begin{tabular}{|c|c|c|c|c|c|c|c|c|}
\hline \multirow{3}{*}{ Variable } & \multicolumn{4}{|c|}{ Overall survival } & \multicolumn{4}{|c|}{ Disease-free survival } \\
\hline & \multicolumn{2}{|c|}{ Univariate } & \multicolumn{2}{|c|}{ Multivariable } & \multicolumn{2}{|c|}{ Univariate } & \multicolumn{2}{|c|}{ Multivariable } \\
\hline & $\begin{array}{l}\text { Hazard ratio } \\
(95 \% \mathrm{CI})\end{array}$ & $p$ value & $\begin{array}{l}\text { Hazard ratio } \\
(95 \% \mathrm{CI})\end{array}$ & $p$ value & $\begin{array}{l}\text { Hazard ratio } \\
(95 \% \mathrm{CI})\end{array}$ & $p$ value & $\begin{array}{c}\text { Hazard ratio } \\
(95 \% \mathrm{CI})\end{array}$ & $p$ value \\
\hline $\begin{array}{l}\text { HR type } \\
\text { negative }\end{array}$ & 1.00 (ref) & & & & 1.00 (ref) & & $\mathrm{NI}$ & \\
\hline $\begin{array}{l}\text { HR type } \\
\text { positive }\end{array}$ & $1.50(0.46-4.92)$ & 0.502 & $\mathrm{NI}$ & & $0.70(0.25-2.00)$ & 0.511 & & \\
\hline \multicolumn{9}{|l|}{ pi6 status } \\
\hline Positive & 1.00 (ref) & & & & 1.00 (ref) & & & \\
\hline Negative & $5.55(1.84-16.72)$ & 0.002 & $\mathrm{NI}$ & & $1.92(0.78-4.73)$ & 0.156 & $\mathrm{NI}$ & \\
\hline Unknown & $2.05(0.70-6.03)$ & 0.192 & & & & & & \\
\hline
\end{tabular}

Multivariable models for both overall survival and disease-free survival were analyzed with stratification of nodal classification (No vs. N1-N3), definite treatment (surgery vs. non-surgery), and type of HPV testing method (liquid microarray vs. DNA chip). HPV, human papillomavirus; HNSCC, head and neck squamous cell carcinoma; CI, confidence interval; NI, not included; ECOG, Eastern Cooperative Oncology Group; PS, performance status; TNM, tumour, node, metastasis; PD, poorly differentiated; HR, high-risk.

${ }^{a}$ Never smokers were included in '<10 pack-year' group.
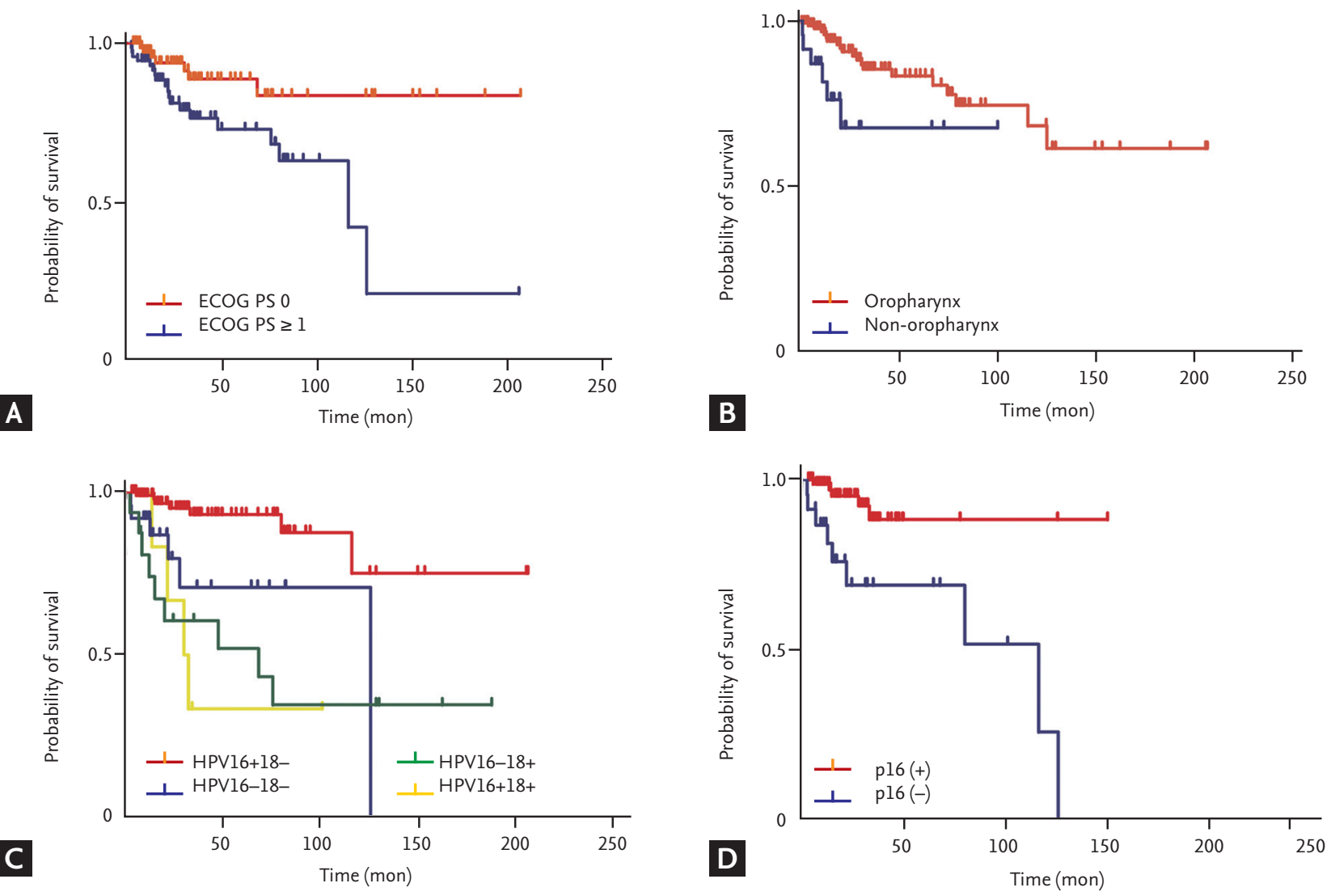

Figure 1. Overall survival of 152 human papillomavirus (HPV)-positive head and neck squamous cell carcinoma patients according to different factors. (A) Eastern Cooperative Oncology Group (ECOG) performance status (PS). (B) Location of primary tumor. (C) HPV genotypes (HPV genotype 16 and 18). (D) p16 status. 

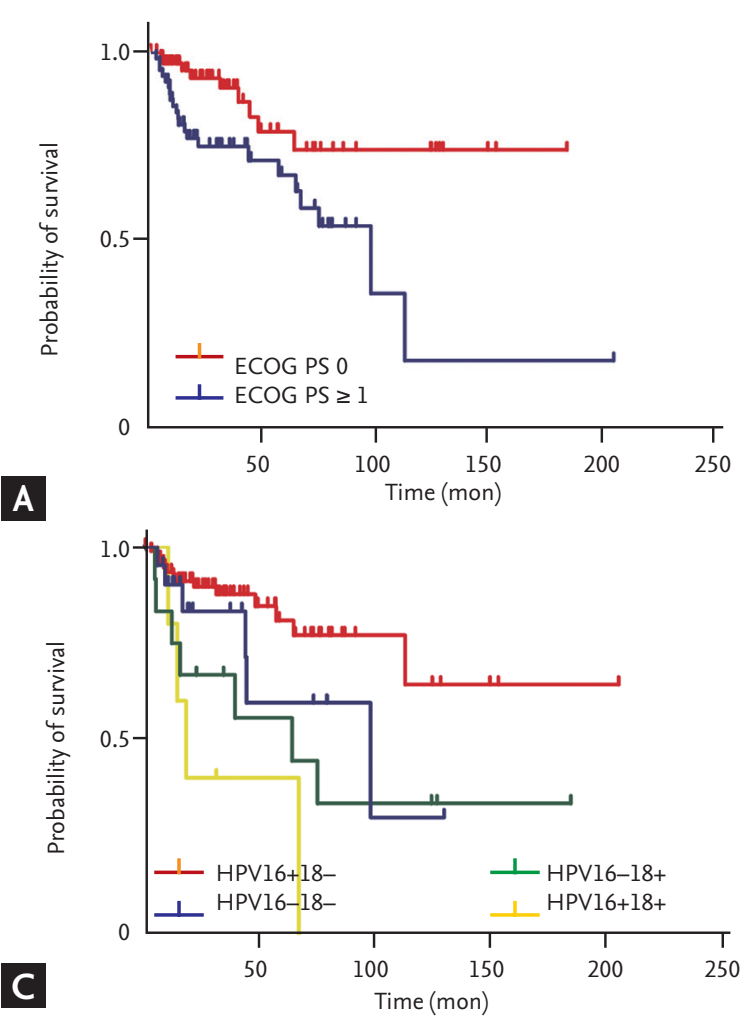

Non-OPC status predicted shorter OS and DFS than OPC in this study. The role of HR HPV in the carcinogenesis of non-OPCs remains unconfirmed [3,13]. A systematic review of the outcomes of patients with HPV-positive non-OPCs found inconsistent survival outcomes [17]. This finding suggests that treatment de-escalation cannot be universally performed for patients with HPV-positive non-OPCs and indicates that HPV infection in nonOPCs may be a "bystander" infection.

Despite the good condition of our patients with HPV-positive HNSCCs overall, the subgroup with ECOG $\mathrm{PS} \geq 1$ had shorter OS and DFS than the subgroup with ECOG PS o. This result is similar to that of a previous study [3].

Interestingly, the findings from our study showed that smoking and drinking were not associated with poorer survival. We suggest two explanations for this result. First, although smoking and alcohol abuse raise the risk of OPC occurrences [18] and additional smoking increases the risk of death in HNSCC patients [3], an association between smoking and HPV has not yet been confirmed. It has been reported previously that HPV positivity is higher in non-smokers than in smokers [19-21]. Second, because of our retrospective study design, limited in-

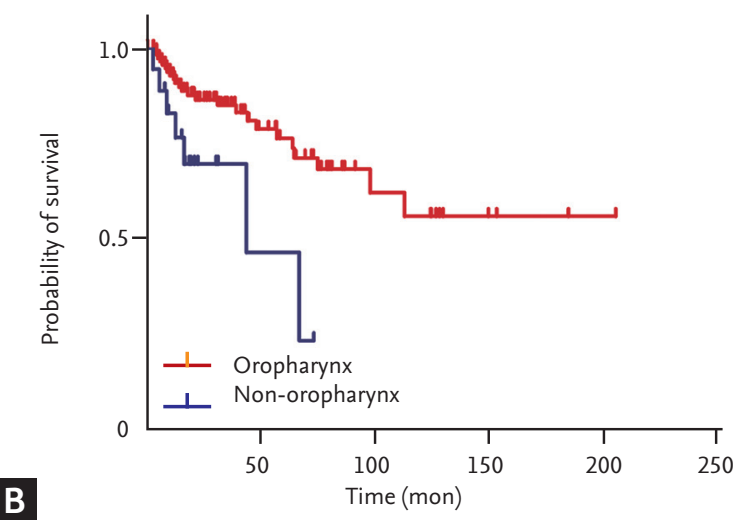

Figure 2. Disease-free Survival of 135 human papillomavirus (HPV)-positive head and neck squamous cell carcinoma patients who received complete response after definitive treatment according to different factors. (A) Eastern Cooperative Oncology Group (ECOG) performance status (PS). (B) Location of primary tumor. (C) HPV genotypes (HPV genotype 16 and 18$)$.

formation about smoking and drinking status were obtained. Further study is needed to determine the effect of smoking and drinking on survival in HPV-positive HNSCCs.

Our study had several limitations. First, because of its retrospective design, we did not have information about p16 expression for many of our study patients. We found that p16 positivity by IHC significantly affected OS but excluded it from the multivariable model because of the high rate of missing results and possibility of collinearity. However, a previous investigation has suggested that the link between p16 positivity by IHC and carcinogenic HPV genotype is not consistent; and some p16-positive tumors by IHC may not be positive for HR HPV [14]. Moreover, p16 expression in non-OPC tumors (oral cavity, hypopharynx, and larynx) is present in only $14 \%$ to $24 \%$ of patients [14]. Therefore, p16 positivity by IHC may be a poor surrogate marker. Second, there may be potential selection bias while capturing the HPV-positive population because in our institution, HPV genotyping methods, which cost approximately $\$ 120$ per test, are not covered by national insurance. Therefore, for 15 years, we did not determine HPV positivity for all head and neck cancer patients. As a result, patients with high- 
er socioeconomic status may be more heavily included in this analysis. However, to the best of our knowledge, other demographic and clinicopathologic factors did not impact whether HPV testing was performed. Third, we used either the DNA chip or liquid bead microarray assay for detecting HPV DNA, and the sensitivity and specificity of these two assays differ. It is possible that these distinct methods may raise selection bias. However, there is no gold-standard method to assess HPV genotypes to date [22]. Moreover, to overcome the possibility of bias, we included the HPV testing method as a stratification variable in the multivariable analysis. Fourth, due to the retrospective design of this study, there might be a bias that results from differing treatment modalities. Although we have attempted to reduce the bias by including definitive treatment as a stratification variable in the analysis, its influence should be considered when interpreting the results. Finally, this study spanned 15 years, during which clinical management of HNSCC likely changed, and this may have influenced the correlates of OS and DFS.

Notwithstanding these limitations, we believe that the OS and DFS of HPV-positive patients who are identified by the present technology can be predicted based on specific clinical factors, and that patients with HPV-positive HNSCCs with unfavorable features are probably not candidates for de-escalation treatment. This study is also a valuable hypothesis-generating study that provides a basis for future in-depth studies investigating the link between HPV genotype 18 and poor prognosis.

In conclusion, HPV-positive HNSCC patients with poor PS and a primary HPV genotype 18-positive tumor located in sites other than the oropharynx may have a poor prognosis. These patients might not be candidates for de-escalation treatment.

\section{KEY MESSAGE}

1. Human papillomavirus (HPV)-positive locally advanced head and neck cancer patients with non-oropharyngeal location, poor performance status, or HPV genotype 18 showed poorer overall survival than those without risk factors.

2. Patients with risk factors might not be candidates for de-escalation treatment.

\section{Conflict of interest}

J. Hun Hah has consulted for Mundipharma Pte Ltd., Singapore, and has received research funding from Ildong Pharmaceutical Co., Ltd., Korea. No other potential competing interest exists.

\section{Acknowledgments}

This study was supported by a grant of the Korea Health Technology R\&D Project "Strategic Center of Cell and Bio Therapy for Heart, Diabetes \& Cancer" through the Korea Health Industry Development Institute (KHIDI), funded by the Ministry of Health and Welfare (MHW), Republic of Korea (grant number: $\mathrm{HI}_{17} \mathrm{C}_{20} \mathrm{O} 5$ ).

\section{REFERENCES}

1. Jung KW, Won YJ, Kong HJ, et al. Cancer statistics in Korea: incidence, mortality, survival, and prevalence in 2012. Cancer Res Treat 2015;47:127-141.

2. Ragin CC, Taioli E. Survival of squamous cell carcinoma of the head and neck in relation to human papillomavirus infection: review and meta-analysis. Int J Cancer 2007;121:1813-1820.

3. Ang KK, Harris J, Wheeler R, et al. Human papillomavirus and survival of patients with oropharyngeal cancer. $\mathrm{N}$ Engl J Med 2010;363:24-35.

4. Mirghani H, Amen F, Blanchard P, et al. Treatment de-escalation in HPV-positive oropharyngeal carcinoma: ongoing trials, critical issues and perspectives. Int J Cancer 2015;136:1494-1503.

5. National Comprehensive Cancer Network. NCCN Guidelines for Head and Neck Cancers Version 2.2016 [Internet]. Plymouth Meeting (PA): NCCN, 2016 [cited 2018 Oct 18]. Available from: https://www.nccn.org.

6. Edge SB; American Joint Committee on Cancer. AJCC Cancer Staging Manual. 7th ed. New York (NY): Springer, 2010.

7. Ock CY, Keam B, Lim Y, et al. Effect of induction chemotherapy on survival in locally advanced head and neck squamous cell carcinoma treated with concurrent chemoradiotherapy: single center experience. Head Neck 2016;38:277-284.

8. Lim Y, Keam B, Koh Y, et al. Clinical outcomes of radiation-based locoregional therapy in locally advanced head and neck squamous cell carcinoma patients not respond- 
ing to induction chemotherapy. Oral Surg Oral Med Oral Pathol Oral Radiol 2013;116:55-60.

9. Eisenhauer EA, Therasse P, Bogaerts J, et al. New response evaluation criteria in solid tumours: revised RECIST guideline (version 1.1). Eur J Cancer 2009;45:228-247.

10. Lee HS, Kim KM, Kim SM, et al. Human papillomavirus genotyping using HPV DNA chip analysis in Korean women. Int J Gynecol Cancer 2007;17:497-501.

11. Choi YD, Jung WW, Nam JH, Choi HS, Park CS. Detection of HPV genotypes in cervical lesions by the HPV DNA Chip and sequencing. Gynecol Oncol 2005;98:369-375.

12. Oh Y, Bae SM, Kim YW, et al. Polymerase chain reaction-based fluorescent Luminex assay to detect the presence of human papillomavirus types. Cancer Sci 2007;98:549-554.

13. Kreimer AR, Clifford GM, Boyle P, Franceschi S. Human papillomavirus types in head and neck squamous cell carcinomas worldwide: a systematic review. Cancer Epidemiol Biomarkers Prev 2005;14:467-475.

14. No JH, Sung MW, Hah JH, et al. Prevalence and prognostic value of human papillomavirus genotypes in tonsillar squamous cell carcinoma: a Korean multicenter study. Cancer 2015;121:535-544.

15. Kim HR, Ha SJ, Hong MH, et al. PD-L1 expression on immune cells, but not on tumor cells, is a favorable prognostic factor for head and neck cancer patients. Sci Rep 2016;6:36956.
16. Bratman SV, Bruce JP, O'Sullivan B, et al. Human papillomavirus genotype association with survival in head and neck squamous cell carcinoma. JAMA Oncol 2016;2:823826 .

17. Isayeva T, Li Y, Maswahu D, Brandwein-Gensler M. Human papillomavirus in non-oropharyngeal head and neck cancers: a systematic literature review. Head Neck Pathol 2012;6 Suppl 1:S104-S12O.

18. Rodriguez T, Altieri A, Chatenoud L, et al. Risk factors for oral and pharyngeal cancer in young adults. Oral Oncol 2004;40:207-213.

19. Fouret P, Monceaux G, Temam S, Lacourreye L, St Guily JL. Human papillomavirus in head and neck squamous cell carcinomas in nonsmokers. Arch Otolaryngol Head Neck Surg 1997;123:513-516.

20. Gillison ML, D'Souza G, Westra W, et al. Distinct risk factor profiles for human papillomavirus type 16-positive and human papillomavirus type 16-negative head and neck cancers. J Natl Cancer Inst 2008;100:407-420.

21. Applebaum KM, Furniss CS, Zeka A, et al. Lack of association of alcohol and tobacco with HPV16-associated head and neck cancer. J Natl Cancer Inst 2007;99:1801-1810.

22. Schache AG, Liloglou T, Risk JM, et al. Evaluation of human papilloma virus diagnostic testing in oropharyngeal squamous cell carcinoma: sensitivity, specificity, and prognostic discrimination. Clin Cancer Res 2011;17:62626271. 